\title{
An siRNA screen identifies transmembrane 7 superfamily member 3 (TM7SF3), a seven transmembrane orphan receptor, as an inhibitor of cytokine-induced death of pancreatic beta cells
}

\author{
A. Beck • R. Isaac • I. Lavelin • Y. Hart • T. Volberg • \\ H. Shatz-Azoulay $\cdot$ B. Geiger $\cdot$ Y. Zick
}

Received: 17 January 2011 / Accepted: 21 June 2011 / Published online: 19 August 2011

(C) Springer-Verlag 2011

\begin{abstract} underlying mechanisms involved in this process. analysed and the corresponding $z$ scores calculated. supplementary material, which is available to authorised users.

A. Beck $\cdot$ R. Isaac $\cdot$ I. Lavelin $\cdot$ Y. Hart $\cdot$ T. Volberg $\cdot$

H. Shatz-Azoulay $\cdot$ B. Geiger $\cdot$ Y. Zick $(\bowtie)$

Department of Molecular Cell Biology,

Weizmann Institute of Science,

Rehovot 76100, Israel

e-mail: yehiel.zick@weizmann.ac.il
\end{abstract}

Aims/hypothesis Pro-inflammatory cytokines induce death of pancreatic beta cells, leading to the development of type 1 diabetes. We sought to identify novel players and the

Methods A high-throughput screen of 3,850 mouse small interfering RNAs (siRNAs) was performed in cytokinetreated MIN6 beta cells. Cells were transfected with the different siRNAs and then treated with a combination of TNF $\alpha$, IL- $1 \beta$ and IFN $\gamma$. Cellular apoptosis (caspase-3/7 activity), and changes in cellular reducing power and cell morphology were monitored. The resulting data were

Results Several gene families were identified as promoting cytokine-induced beta cell apoptosis, the most prominent being those encoding ubiquitin ligases and serine/threonine kinases. Conversely, deubiquitinating enzymes appeared to reduce apoptosis, while protein phosphatases were mainly associated with lowering cellular reducing power. The screen suggested with high confidence the involvement of several novel genes in cytokine-induced beta cell death, including Camkk2, Epn3, Foxp3 and Tm7sf3, which encodes an orphan seven transmembrane receptor. siRNAs to $\operatorname{Tm} 7 s f 3$ promoted cytokine-induced death of MIN6 cells and human pancreatic islets, and abrogated insulin secretion

Electronic supplementary material The online version of this article (doi:10.1007/s00125-011-2277-3) contains peer-reviewed but unedited in these cells. These findings implicate transmembrane 7 superfamily member 3 as a potential new player in the inhibition of cytokine-induced death and in the promotion of insulin secretion from pancreatic beta cells.

Conclusions/interpretation The signalling pathways and novel genes that we identified in this screen and that mediate beta cell death offer new possible targets for therapeutic intervention in diabetes and its adverse complications.

Keywords Pancreatic beta cells · Pro-inflammatory cytokines $\cdot$ siRNA screens $\cdot$ TM7SF3

$\begin{array}{ll}\text { Abbreviations } \\ \text { cIAP } & \text { Cellular inhibitor of apoptosis protein } \\ \text { CTB } & \text { CellTiter Blue } \\ \text { 3D } & \text { Three-dimensional } \\ \text { DISC } & \text { Death-inducing signalling complex } \\ \text { FADD } & \text { Fas (TNFRSF6)-associated via death domain } \\ \text { GSIS } & \text { Glucose-stimulated insulin secretion } \\ \text { HTS } & \text { High-throughput screen } \\ \text { IKK } & \text { IkB kinase } \\ \text { JNK } & \text { JUN N-terminal kinase } \\ \text { NF-kB } & \text { Nuclear factor kB } \\ \text { siRNA } & \text { Small interfering RNA } \\ \text { TM7SF3 } & \text { Transmembrane 7 superfamily member } 3\end{array}$

\section{Introduction}

Islet transplantation is the only current treatment for type 1 diabetes that brings about insulin independence [1]. Over time, however, these islet allografts deteriorate, usually within 2 to 5 years following transplantation, with patients 
returning to insulin dependence [1]. Treatment failure is primarily attributed to inflammatory reactions triggered by cytokines and free radicals capable of inflicting severe beta cell damage [2]. Pro-inflammatory cytokines such as IL-1ß, $\mathrm{TNF} \alpha$ and IFN $\gamma$ induce beta cell death through production of the transcription factors nuclear factor $\mathrm{kB}(\mathrm{NF}-\mathrm{kB})$ and signal transducer and activator of transcription 1 [3]. The latter acts through the activation of JUN N-terminal kinase (JNK) [3], whereas NF-KB activation promotes production of nitric oxide and a variety of inflammatory chemokines, as well as depletion of calcium from the endoplasmic reticulum, thus inducing beta cell death by apoptotic and non-apoptotic processes [3].

Microarray experiments have revealed that $\sim 1,500$ genes are up- or downregulated in beta cells after exposure to different combinations of cytokines, illustrating the large number of potential players in the process leading to beta cell death [4-6]. However, microarray studies have not been able to discriminate between genes that induce beta cell death and those that are up- or downregulated as a consequence of the death process. The complexity of the system is further illustrated by the fact that exposure of beta cells to IL-1 $\beta$ alone is insufficient to induce cell death and that only when IL-1 $\beta$ is combined with IFN $\gamma$ does significant cell death occur [7]. Inflammatory responses lead to the activation of cytokine receptors, recruitment of adapter molecules and formation of the death-inducing signalling complex (DISC), which, in turn, triggers apoptosis [8]. Nevertheless, despite the well documented involvement of apoptosis in beta cell death [3, 9], alternative death mechanisms such as necrosis [10] have also been implicated in this process [11].

In the present study we conducted a high-throughput screen (HTS) for the effects of specific small interfering RNAs (siRNAs) [12] with a view to identifying novel players involved in the induction of cytokine-induced death of pancreatic beta cells and exploring the underlying mechanisms involved. Screening of siRNA libraries has become an increasingly effective approach in functional genomics [13], leading to the identification of novel genes involved in diverse cellular processes such as tumour suppression, cell proliferation, apoptosis, proteasome and p53 functions, adhesion formation and NF-KB activation [14]. These rather promising studies provided the incentive for the current study, in which MIN6, an established glucose-responsive and insulinsecreting mouse beta cell line [15], was used to search for novel genes involved in cytokine-induced beta cell death.

\section{Methods}

Cytokines The cytokine mixture (Cytomix) consisted of $3 \mathrm{nmol} / 1 \mathrm{TNF} \alpha$ (Prospec-Tany Technogene, Rehovot,
Israel), $3 \mathrm{nmol} / 1$ IFN- $\gamma$ and $1.5 \mathrm{nmol} / 1$ IL- $1 \beta$ (MD Biosciences, Ness Ziona, Israel). Their biological activities were 10 units/ng (TNF $\alpha$, IFN- $\gamma)$ and 200 units/ng (IL-1 $\beta$ ).

Isolation of murine islets Digested pancreases were filtered through 1,000 and $500 \mu \mathrm{m}$ sieves, and islets $>75$ and $<250 \mu \mathrm{m}$ were handpicked under a stereoscope. Islets were cultured in RPMI 1640 medium containing $5 \mathrm{mmol} / \mathrm{l}$ glucose, $10 \%$ (vol./vol.) FCS, 2 mmol/l L-glutamine, 100 units $/ \mathrm{ml}$ penicillin, $100 \mu \mathrm{g} / \mathrm{ml}$ streptomycin and $40 \mu \mathrm{g} / \mathrm{ml}$ gentamycin, and were used within $48 \mathrm{~h}$ of isolation.

Culture of human islets Isolated human islets ( $>90 \%$ purity) were provided by the European Consortium for Islets Transplantation (ECIT; Islet for Basic Research programme; http://ecit.dri-sanraffaele.org/en/islet-transplatntation/index. html) through a Juvenile Diabetes Research Foundation award $31-2008-413$. Islets were cultured at $37^{\circ} \mathrm{C}$ in a $5 \%$ $\mathrm{CO}_{2}$ humidified atmosphere in CMRL 1066 medium containing $10 \%$ (vol./vol.) FBS, $2 \mathrm{mmol} / \mathrm{l} \mathrm{L}$-glutamine, 100 units $/ \mathrm{ml}$ penicillin, $100 \mu \mathrm{g} / \mathrm{ml}$ streptomycin, $0.25 \mu \mathrm{g} / \mathrm{ml}$ amphotericin and $40 \mu \mathrm{g} / \mathrm{ml}$ gentamycin. The medium was changed every other day. Human islets were dispersed by 4 min incubation at $37^{\circ} \mathrm{C}$ with trypsin/EDTA. Trypsinised islets were washed with cold CMRL 1066 medium containing $10 \%$ (vol./vol.) FBS, gently pipetted and re-suspended in CMRL 1066 containing 10\% (vol./vol.) FBS. Human islets studies received Ethics Committee approval.

Mice Male C57BL/6 J mice (Harlan Laboratories, Rehovot, Israel) (aged 8-10 weeks) were housed under standard light/dark conditions with free access to food and water. Experiments were approved by the Animal Care and Use Committee of the Weizmann Institute of Science.

High-throughput screening High-throughput screens were carried out, as detailed in the electronic supplementary material (ESM), using selected siRNA SMARTpool libraries purchased from Dharmacon (Lafayette, CO, USA). Altogether, these libraries targeted 3,850 non-redundant mouse genes (ESM Table 1). Each SMARTpool contained four different siRNA duplexes targeting a particular mRNA. Using a robot (BIOMEK FX; Beckman-Coulter, Brea, CA, USA), four aliquots of each siRNA SMARTpool were transferred into four 384-well plates to reach a final siRNA concentration of $25 \mathrm{nmol} / \mathrm{l}$ per well. To avoid potential bias caused by a given well position, each aliquot was placed in a different location in the four plates. Thus each siRNA was screened as four independent 'biological replicates', augmenting the reliability of the screen.

Other methods Further details on materials, cells, assays of glucose-stimulated insulin secretion (GSIS) and caspase-3/7 
activity, cellular reducing power, quantitative RT-PCR, immunofluorescence and live cell imaging are available in the ESM.

\section{Results}

Effects of pro-inflammatory cytokines on beta cell viability Isolated pancreatic islets are the physiological system of choice to screen for siRNAs of genes associated with cytokine-induced beta cell death; however, their heterogeneity makes them less than optimal for large-scale HTS of siRNAs. As an alternative, we carried out a screen using the MIN6 insulin-secreting beta cell line. As shown in ESM Fig. 1, insulin secretion from MIN6 cells treated with $20 \mathrm{mmol} / \mathrm{l}$ glucose was sixfold higher than basal secretion at $0.3 \mathrm{mmol} / \mathrm{l}$ glucose. We thus confirmed previous findings [15] and concluded that our line of MIN6 cells was indeed glucose-responsive and appropriate for further experimentation.

Cytokines induce beta cell death by means of apoptotic and non-apoptotic mechanisms [16]. To characterise the apoptotic effects of cytokines, MIN6 cells were incubated with a combination of $3 \mathrm{nmol} / \mathrm{TNF} \alpha, 3 \mathrm{nmol} / \mathrm{l} \mathrm{IFN}-\gamma$ and $1.5 \mathrm{nmol} / \mathrm{l} \mathrm{IL}-1 \beta$ (Cytomix) for varying periods of time. The optimum time-window for siRNA expression (48-72 h after transfection) was taken into account; thus, the maximal feasible time for Cytomix stimulation (beginning $24 \mathrm{~h}$ after transfection) could not have been longer than $48 \mathrm{~h}$. As shown in ESM Fig. 2, high levels of MIN6 cell death were achieved when cells were treated with Cytomix for $48 \mathrm{~h}$. This was evident from the increased caspase-3/7 activity (ESM Fig. 2a), lower reducing power (CellTiter Blue [CTB] assay; ESM Fig. 2b) and changes in cell morphology (ESM Fig. 2c).

Cellular reducing power largely correlates with cellular ATP levels; the higher the reducing power, the higher the rate of ATP production [17]. Non-apoptotic beta cell death is linked to a decrease in cellular reducing power, which results in a decline in cellular ATP levels [18]. To assess the relative contribution of apoptotic vs non-apoptotic aspects of cytokine-induced death, MIN6 cells were treated with cytokines in the presence of the caspase inhibitor (zVADfmk) or an inhibitor (necrostatin-1) of receptor-interacting protein 1, a key enzyme in the necrotic process [10]. Inclusion of zVAD-fmk effectively inhibited ( 80\%) cytokine-induced activation of casapse-3/7 in MIN6 cells (ESM Fig. 3) and resulted in partial protection (45-60\%) of MIN6 cells (ESM Fig. 4a) and primary murine islets (ESM Fig. 4b) from a decrease in their reducing power induced by cytokines. Necrostatin-1 was at least as effective as zVADfmk in conferring protection from cytokine-induced death of MIN6 cells $(\sim 50 \%)$ or mouse islets $(\sim 100 \%$; ESM Fig. 4). These findings suggest that cytokines induce apoptotic and necrotic forms of death, with different contributions in MIN6 cells and primary murine islets.

High-throughput screening for modulators of cytokineinduced beta cell death High-throughput screens for regulators of cytokine-induced beta cell death were carried out using selected siRNA libraries (Dharmacon). The libraries (ESM Table 1) were chosen on the basis of a possible involvement of their target genes in the modulation of cytokine-induced beta cell death. They included siRNAs that target protein kinases, phosphatases, ubiquitin ligases, deubiquitinating enzymes, receptors, ion channels, transcription factors, etc. Altogether, these libraries targeted 3,850 non-redundant mouse genes. The libraries were screened for their effects on caspase-3/7 activity, cellular reducing power determined by CTB fluorometric assay (Promega, Madison, WI, USA) and morphological changes in MIN6 cells treated with cytokines (Fig. 1). siRNA 'hits' with $z$ scores $\geq 2.0$ in these screens are listed in ESM Table 2. The screens identified a number of siRNAs that target genes previously unknown to be involved in cytokine-induced beta cell death, along with genes known as mediators of this process. The latter included caspase-3

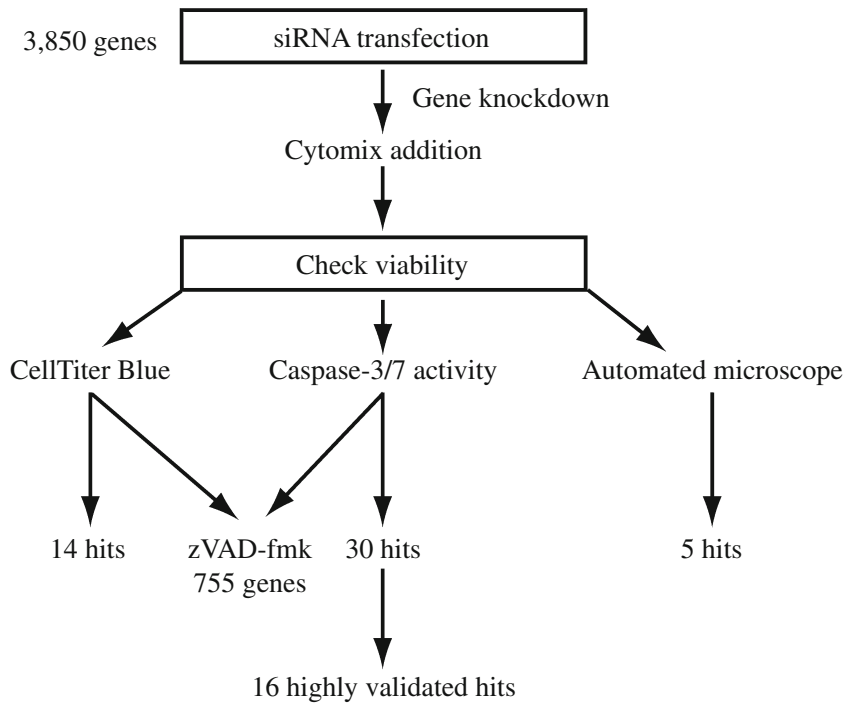

Fig. 1 Schematic representation of the HTSs. Individual siRNAs $(n=3,850)$ were transfected into MIN6 cells, which were further treated with cytokines. The effects of the siRNAs were analysed using three different HTS techniques: (1) a CTB screen, which measures cellular reducing power; (2) caspase-3/7 activity screen, as a measure of apoptosis; and (3) an automated microscopy screen to identify changes in cell morphology. A total of 755 selected siRNAs were transfected into cells treated with cytokines and zVAD-fmk, and subjected to HTS using CTB. The different screens yielded the indicated number of potential 'hits', determined as described above (Methods). In the caspase-3/7 screen 30 'hits' had $z$ scores $>2$ (see ESM Table 2) and were subjected to validation. Of those, 16 (listed in Table 1) were found to be highly validated 
itself, as well as siRNAs to Tial (which encodes an apoptosis-promoting factor) [19], Pou $4 f 2$ (which enhances the pro-apoptotic effects of p53) [20] and $L m x 1 b$ [21], which regulates the expression of NF- $\mathrm{kB}$ target genes, a key element in the induction of beta cell apoptosis [22]. Similarly, Atg4b [23] scored high in the CTB screen.

Distribution of apoptosis-inducing genes in individual libraries To evaluate the distribution of apoptosisinducing genes in the different libraries, each library was divided into ten bins, each representing a range of $z$ scores. The width of each bin represented the fraction of siRNAs that had $z$ scores within an indicated range (e.g. $z$ scores 1.0-1.5 in the sixth bin; Fig. 2). As expected, the library of siRNAs related to cytokine receptors included the largest fraction of pro-apoptotic genes (with siRNAs having high negative $z$ scores; the 'Dark red' library). The library of ubiquitin ligases was also highly enriched in pro-apoptotic genes. Conversely, the library of deubiquitinating enzymes included the largest fraction of anti-apoptotic genes (siRNAs with high positive $z$ scores; the 'Orange' library).

Comparison of libraries promoting apoptotic vs nonapoptotic beta cell death Apoptotic cell death requires high cellular ATP levels, while non-apoptotic (e.g. necrotic) cell death is characterised by a reduction in cellular ATP content [24]. To identify gene families associated with these processes, we compared the results of the screens in which cellular reducing power and caspase-3/7 activity were analysed. The list of $3,850 z$ scores from each screen was limited to a threshold that included only $z$ scores in the upper $25 \%$ top or bottom fraction of the entire population. Based on this criterion, siRNAs were divided into four categories (Fig. 2): (1) low caspase/high CTB $z$ scores, representing siRNAs to pro-apoptotic and pro-necrotic genes; (2) high caspase/high CTB $z$ scores, i.e. siRNAs to anti-apoptotic and pro-necrotic genes; (3) low caspase-3/ low CTB $z$ scores, i.e. siRNAs to pro-apoptotic and antinecrotic genes; and (4) high caspase-3/low CTB $z$ scores, representing siRNAs to anti-apoptotic and anti-necrotic genes.

Next, the distribution of siRNAs that corresponded to the above criteria within individual libraries was determined. As shown in Fig. 2, the library of deubiquitinating enzymes was enriched in siRNAs to anti-apoptotic and anti-necrotic genes, whereas the libraries of cytokine receptors and ubiquitin ligases were enriched in siRNAs to pro-apoptotic genes. Similarly, the library of protein phosphatases was enriched in pro-necrotic genes, while the family of protein kinases was enriched in pro-apoptotic genes. These results suggest that the ubiquitin system and kinases/phosphatases are highly involved in regulating cytokine-induced beta cell death. a
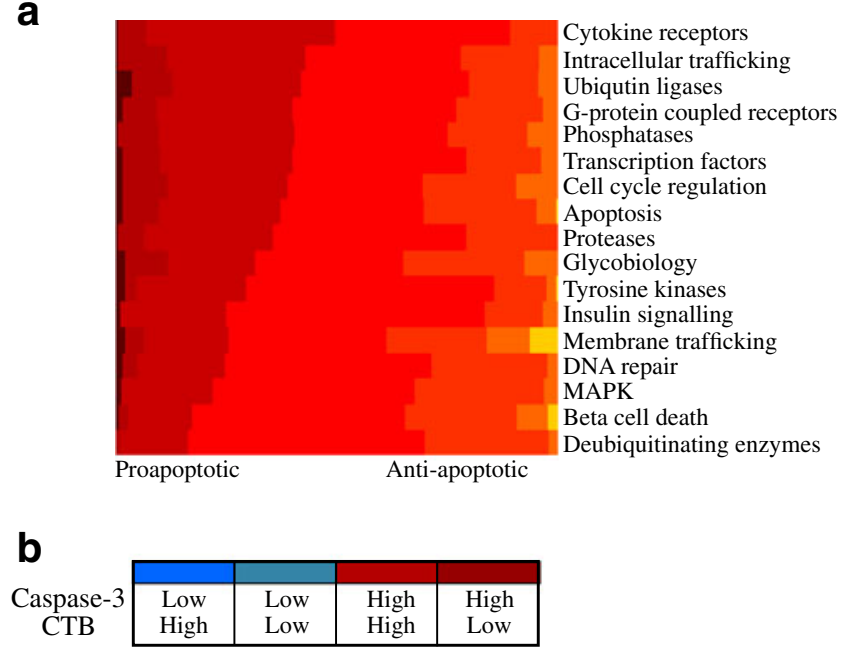

C

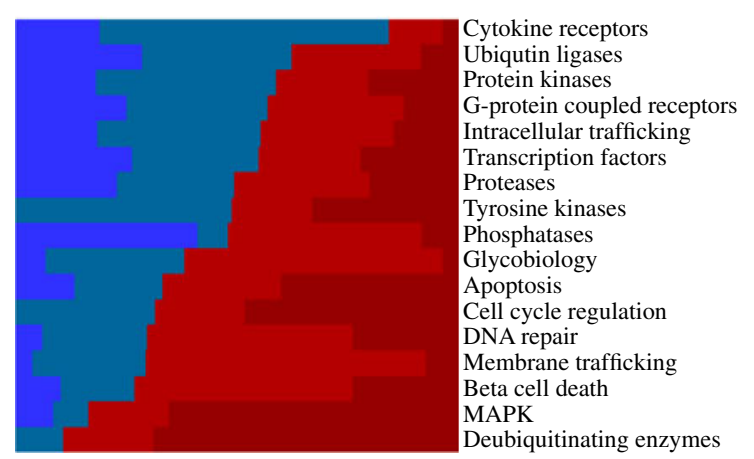

Fig. 2 Distribution of $z$ scores in individual libraries. a The sorted $z$ scores of 3,850 siRNAs that were screened for caspase activity were divided into ten bins, each representing a range of $z$ scores from lowest to highest, using MATLAB R2007a (http://www.mathworks. com/help/toolbox/compiler/rn/bq249ht.html). The distribution of $z$ scores in individual libraries were then calculated. The width of each bin represents the fraction of siRNAs with $\mathrm{z}$ scores of the indicated range. Dark red, lowest $z$ scores; yellow, highest $z$ scores. b The threshold of the sorted $z$ scores of the 3,850 siRNAs that had been screened for caspase activity (apoptosis) or cellular reducing power (CTB) was set to include only $z$ scores falling within the upper $25 \%$ top or bottom fraction of the population. Based on these criteria, the siRNAs were divided into four categories as indicated. c The fraction of siRNAs with $z$ scores within individual libraries was determined. The libraries were then sorted according to their apoptotic or non-apoptotic (e.g. necrotic) features. The colours correspond to the criteria indicated in $\mathbf{b}$

Validation of novel 'hits' Several unknown genes (ESM Table 2) were identified as potential 'hits'. To validate these 'hits', 14 'pro-apoptotic' and 16 'anti-apoptotic' high-score hits (determined by caspase-3/7 activity) were re-screened using four individual siRNAs for each gene. Hits in which at least two of the four siRNAs yielded an effect similar to that of the SmartPool were considered high-confidence hits, and 16 'hits' scored positive by this criterion, while seven 'hits' even scored positive in three (of four) individual siRNAs (Table 1). Many of these hits, such as $\operatorname{Tm} 7 s f 3$ 
Table 1 List of highly validated hits with $z$ score $>2.0$, where at least two of the four single siRNAs yielded a similar effect to that of the SmartPool

\begin{tabular}{lclc}
\hline Symbol & Scores (of 4) & Accession & Caspase-3/7 (mean \pm SD) \\
\hline E130008b10rik & 2 & E130008B10RIK**NM_207238 & $-3.0 \pm 1.0$ \\
Gyk & 2 & GYK**NM_008194 & $-2.6 \pm 0.7$ \\
Psma2 & 2 & PSMA2**NM_008944 & $-2.3 \pm 0.9$ \\
Ndfip1 & 3 & NDFIP1**XM_128893 & $-2.2 \pm 0.8$ \\
Lmxlb & 2 & LMX1B**NM_010725 & $-2.1 \pm 1.1$ \\
Alx3 & 3 & ALX3**NM_007441 & $-1.9 \pm 0.8$ \\
Tcfeb & 3 & TCFEB**NM_011549 & $-1.9 \pm 0.7$ \\
Dgkq & 3 & DGKQ**NM_199011 & $-1.9 \pm 0.2$ \\
Pou4f2 & 2 & POU4F2**NM_138944 & $-1.8 \pm 1.1$ \\
Foxp3 & 2 & FOXP3**NM_054039 & $1.8 \pm 0.5$ \\
Epn3 & 2 & EPN3**NM_027984 & $2.1 \pm 1.0$ \\
D9bwg0185e & 2 & D9BWG0185E**NM_173781 & $2.2 \pm 1.1$ \\
Stk22s $1^{\mathrm{c}}$ & 2 & STK22S1**NM_011651 & $2.3 \pm 0.8$ \\
Jak2 & 2 & JAK2**NM_008413 & $2.4 \pm 1.1$ \\
Camkk2 & 2 & CAMKK2**NM_145358 & $2.5 \pm 1.1$ \\
Tm7sf3 & 3 & TM7SF3**XM_132970 & $2.7 \pm 1.1$ \\
\hline
\end{tabular}

(which encodes a seven transmembrane superfamily receptor 3) [25] or Epn3, were not previously associated with cytokine-induced beta cell death.

Tm7sf3-specific siRNA effectively reduces endogenous levels of $T m 7 s f 3 \quad m R N A$ The potency of the siRNAs targeting $T m 7 s f 3$ to reduce its endogenous mRNA levels was evaluated next. We found that three of four individual siRNAs that constituted the siRNA SmartPool of Tm7sf3 effectively reduced its endogenous mRNA levels in MIN6 cells by $\sim 80 \%$ (ESM Fig. 5). These effects were specific because non-targeting siRNAs, which served as control, did not have any effect. Hence, the SmartPool siRNA of $T m 7 s f 3$ could be considered an efficient tool for reducing $T m 7 s f 3$ mRNA levels.

Effects of suppression of TM7SF3 on the morphology and cytoskeletal organisation of cytokine-treated MIN6 cells The siRNA screen implicated transmembrane 7 superfamily member 3 (TM7SF3) as an inhibitor of cytokine-induced death of MIN6 cells. To gain further insights into its mode of action, we examined the effects of $T m 7 s f 3$ siRNAs on cellular morphology and dynamics, as well as the cytoskeletal and nuclear organisation of cytokine-treated MIN6 cells. Cells were transfected with $T m 7 s f 3$ siRNAs, treated with Cytomix, and examined by time-lapse and 3-dimensional (3D) fluorescence microscopy.

The analysis showed that after $\sim 5 \mathrm{~h}$ of Cytomix treatment, the flat epithelial MIN6 (control) islands tended to undergo apparent clustering and rounding-up into $3 \mathrm{D}$ aggregates, although the individual cells within these colonies showed only mild effects on their nuclei or cytoskeletal systems; these effects were characterised by somewhat disorganised microtubules (not shown), possibly attributable to cell contraction following treatment [26]. Longer incubation (e.g. $8 \mathrm{~h}$ ) in the presence of cytokines (Fig. 3a) was characterised by progressive cell death and detachment from the substrate, while addition of zVAD-fmk largely arrested this clustering process. Knockdown of $T m 7 s f 3$ resulted in apparent cell rounding and clustering, which occurred independently of the addition of cytokines, with cytokine treatment having only limited effect on these cells. Interestingly, zVAD-fmk induced spreading of these cells, resulting in the formation of flat cellular islands, similar to cells transfected with non-targeting (control) siRNAs.

To further characterise the effects TM7SF3, MIN6 cells were transfected with $25 \mathrm{nmol} / 1$ of non-targeting or Tm $7 \mathrm{sf3}$ specific siRNAs. At $48 \mathrm{~h}$ post-transfection, cells were treated for $1 \mathrm{~h}$ with $20 \mu \mathrm{mol} / \mathrm{l} \mathrm{zVAD}-\mathrm{fmk}$, prior to addition of cytokines for $24 \mathrm{~h}$. Cells were then fixed and subjected to triple-labelling for actin, microtubules and DNA. The labelled cells were subjected to 3D deconvolution fluorescence microscopy. Examination of cytokine-treated cells (ESM Fig. 6) confirmed that this treatment induced cell aggregation into 3D colonies, characterised by a marked thickening of the cell layer. Addition of zVAD-fmk somewhat blocked this process. In contrast, knockdown of the anti-apoptotic gene $\operatorname{Tm} 7 s f 3$ induced aggregation into 3D colonies even in the absence of cytokines. The microtubule system in $T m 7 s f 3$ knocked-out cells was disorganised, this disorganisation being characterised by the tendency of microtubules to aggregate. Normal microtubule organisation was partially restored by zVAD-fmk treatment (not 
Fig. 3 Effects of siRNA to $T m 7 s f 3$ on MIN6 cells. MIN6 cells were transfected with the indicated siRNAs for $48 \mathrm{~h}$ and treated as detailed. a Cells were treated with Cytomix with or without zVAD-fmk for $8 \mathrm{~h}$, and then fixed and labelled with fluoresceinated antibodies against tubulin (red), FITC-phalloidin (green) and DAPI (blue) for DNA labelling. Images are of representative cells from two independent experiments. NT, not treated. b Cells were left untreated (white bars) or were treated with Cytomix (black bars) for $6 \mathrm{~h}$, and then assayed for caspase activity. NonT, non-targeting. c Cells were left untreated or were treated with Cytomix for $72 \mathrm{~h}$. Cellular reducing power was assayed using a CTB kit. d-f Cells were left untreated or were treated with Cytomix for $24 \mathrm{~h}$, then stained with propidium iodide (PI) and annexin V, followed by FACS analysis. Outputs were gated for live cells (R1), annexin V-positive cells (R2) and PI-positive cells (R3), and quantified. Results $(\mathbf{b}-\mathbf{f})$ are mean \pm SEM of three independent experiments; $* p<0.05, * * p<0.01$ and $* * * p<0.001$ vs non-targeting siRNA assayed under the same conditions

shown). These findings suggest that TM7SF3 might promote cell death even in a cytokine-independent manner.

Tm7sf3 inhibits cytokine-mediated induction of caspase activity in MIN6 cells To study the mechanism underlying the above findings, MIN6 cells were transfected with control non-targeting siRNAs or with $T m 7 s f 3$ siRNAs, prior to stimulation with Cytomix. Treatment with Cytomix for $6 \mathrm{~h}$ significantly increased caspase activity in nontransfected cells and cells expressing non-targeting siRNAs (Fig. 3b). A larger increase, mainly in cytokine-treated cells, was observed in cells transfected with $T m 7 s f 3$ siRNAs, suggesting that TM7SF3 inhibits cytokineinduced activation of caspase-3/7. As expected, transfection of siRNAs to caspase-3/7 abrogated the increase in caspase activity (not shown).

TM7SF3 helps maintain cellular reducing power in MIN6 cells To further analyse the molecular processes associated with the protective effects of TM7SF3, MIN6 cells were incubated with non-targeting siRNAs or with $T m 7 s f 3$ siRNAs prior to stimulation for $72 \mathrm{~h}$ with Cytomix. $T m 7 s f 3$ siRNAs induced a $40 \%$ lowering in the reducing power of non-treated cells, when compared with cells expressing non-targeting siRNAs (Fig. 3c). These findings suggest that transfection as such lowers the reducing power of MIN6 cells even in the absence of cytokines, and that TM7SF3 plays a role in preventing this process. Indeed, the reducing power of cells expressing non-targeting siRNAs was $\sim 25 \%$ lower than that of naive non-treated cells. Treatment with cytokines lowered by $\sim 45 \%$ the reducing power of cells expressing non-targeting siRNAs, while a larger reduction in cell viability was observed in cells expressing $T m 7 s f 3$ siRNAs (Fig. 3c). These findings (Fig. 3b, c) support the hypothesis that TM7SF3 promotes cell survival in the presence or in the absence of cytokines, and has a larger impact in cytokine-treated cells. Conversely, its siRNAs promote apoptotic as well as non-apoptotic cell death. This
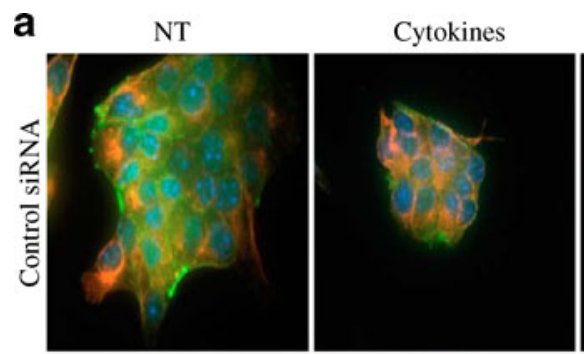

Cytokines $+z V A D$
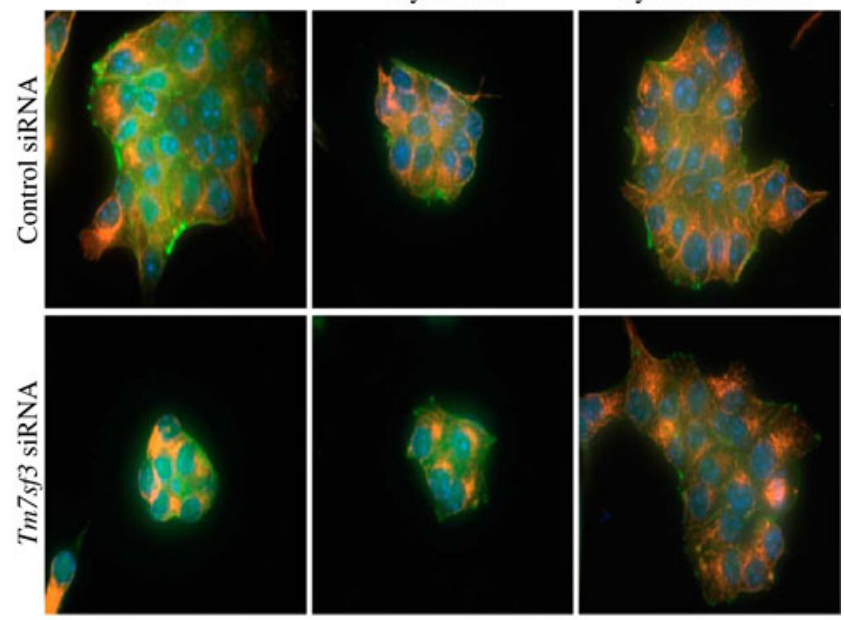

b

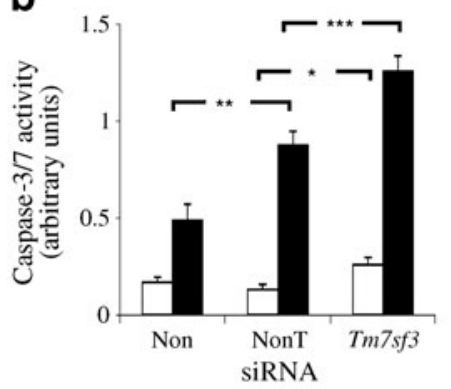

C

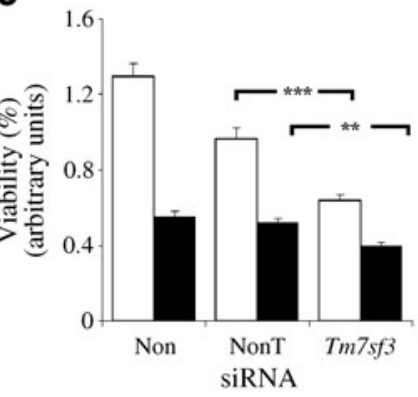

d
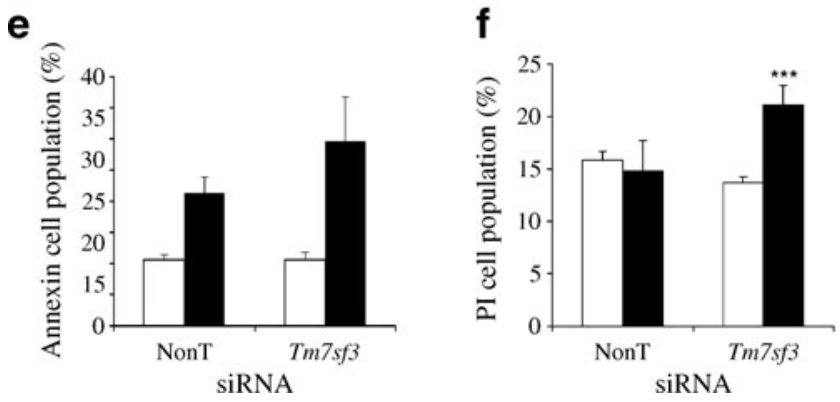

conclusion was substantiated by FACS analysis (Fig. 3d-f), where we demonstrated that $T m 7 s f 3$ siRNAs significantly reduced the fraction of viable cells treated with cytokines (Fig. 3d). The fraction of apoptotic (annexin V-positive) cells treated with $\operatorname{Tm} 7 s f 3$ siRNAs was somewhat increased 
(not significantly; Fig. 3e), while the fraction of necrotic (propidium iodide-positive) cells that were treated with cytokines was significantly increased in the presence of $T m 7 s f 3$ siRNAs (Fig. 3f). Tm $7 s f 3$ siRNAs had no effect on cells treated in the absence of cytokines (Fig. 3d-f). Interestingly, treatment with cytokines mainly increased the population of annexin V-positive cells (Fig. 3e) with little effects on the population of propidium iodide-positive cells treated with control siRNAs (Fig. 3f).

Effects of TN7SF3 on insulin secretion Insulin secretion is tightly regulated by and is induced upon an increase in cellular ATP [27]. Therefore, the effects of TM7SF3 on insulin secretion were studied. Human pancreatic islets were used, being the most physiological model system. First, the potency of siRNAs targeting TM7SF3 to reduce its endogenous mRNA levels in human islets was evaluated. As shown in Fig. 4a, TM7SF3 siRNAs effectively reduced the endogenous mRNA levels of TM7SF3 in human islets by $\sim 75 \%$. Next, the effects of TM7SF3 siRNAs on insulin secretion were studied. As shown in Fig. 4b, GSIS in dispersed human islets was inhibited by $\sim 30 \%$ in islets treated with TM7SF3 siRNAs, when compared with islets treated with non-targeting siRNAs. Furthermore, while inclusion of cytokines inhibited GSIS by $30 \%$ in islets treated with non-targeting siRNAs, cytokines inhibited the already reduced GSIS in islets treated with $T M 7 S F 3$ siRNAs by $50 \%$. These findings suggest that TM7SF3 helps maintain insulin secretion in human islets as part of its action as an anti-apoptotic protein that keeps maintaining high cellular reducing power and ATP levels. This conclusion was supported by the finding that cytokine-induced apoptosis in human islets (Fig. 4c) was significantly increased in cells expressing $T M 7 S F 3$
siRNAs, when compared with cells expressing control siRNAs. A small, yet significant increase in apoptosis was also detected in cells treated in the absence of cytokines.

Effects of TM7SF3 on the extrinsic apoptotic pathway Beta cell death induced by cytokines (TNF $\alpha$, IL-1 $\beta$ and IFN $\gamma$ ) involves several signalling pathways [3]. For example, binding of TNF $\alpha$ to its receptor involves generation of a DISC and activation of caspase-8 [28]. To determine whether TM7SF3 directly affects these signalling pathways, its effects on Fas (TNFRSF6)-associated via death domain (FADD), FAS and caspase- 8 were analysed. Introduction of $T m 7 s f 3$ siRNAs increased the cellular content of FADD in nontreated cells by $30 \%$; this increase was significantly greater (3.5-fold) in cells treated with TNF $\alpha$ for $5-12$ h (Fig. 5a). The effects of the $T m 7 s f 3$ siRNAs diminished by $24 \mathrm{~h}$, mainly due to the increase in FADD content in cells transfected with control siRNAs. A transient, modest increase in FAS (which mostly did not reach statistical significance) was also observed (Fig. 5a). $T m 7 s f 3$ siRNAs also induced a significant increase in the cellular content of pro-caspase-8 in cells treated with Cytomix (Fig. 5b). A small (but not significant) increase was observed in cells treated only with TNF $\alpha$ (Fig. 5b). These results suggest that, in accordance with its anti-apoptotic function, TM7SF3 reduces the cellular content of FADD and pro-caspase- 8 in control, and even more so in cytokine-treated cells.

\section{Discussion}

A HTS of 3,850 mouse siRNAs was used to identify novel genes mediating cytokine-induced death of pancreatic beta cells. The screens revealed the involvement of several novel a

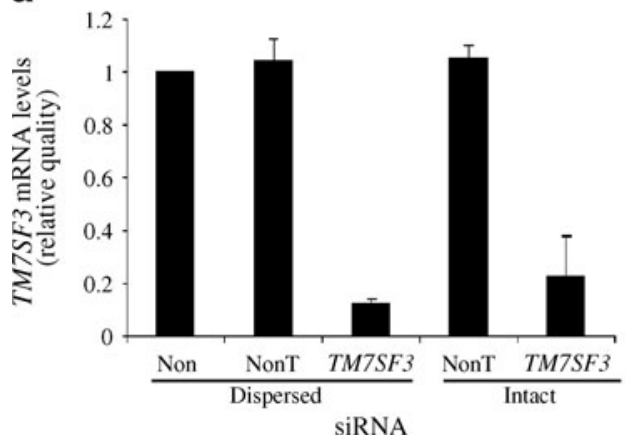

b

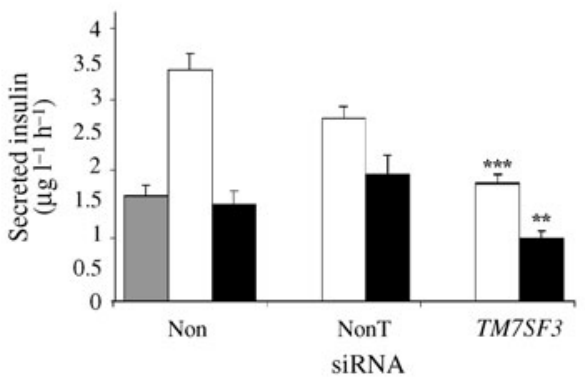

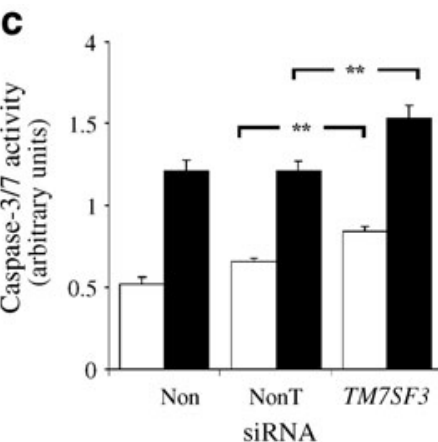

Fig. 4 Effects of TM7SF3 siRNA on caspase activity and insulin secretion in intact human islets treated with cytokines. Intact human islets were transfected for $48 \mathrm{~h}$ with $25 \mathrm{nmol} / 1$ of the indicated siRNAs. a RNA was extracted and quantified by quantitative RTPCR. Non, untreated; NonT, non-targeting. b Islets were left untreated (grey and white bars) or were treated with Cytomix (black bars) for $16 \mathrm{~h}$. Islets were then incubated in KRBH buffer containing $3.3 \mathrm{mmol} / 1$ glucose (grey bars) or $16.7 \mathrm{mmol} / 1$ glucose (white bars, black bars). The insulin concentration in the culture medium was determined and normalised per total insulin content. $\mathbf{c}$ Islets were left untreated (white bars) or were treated with threefold concentrated Cytomix (black bars) for $24 \mathrm{~h}$, and then assayed for caspase activity. Data are the mean \pm SEM of three $(\mathbf{b})$ and two $(\mathbf{a}, \mathbf{c})$ independent experiments, respectively; $* * p<0.01$ and $* * * p<0.001$ vs nontargeting siRNAs assayed under the same conditions 
a

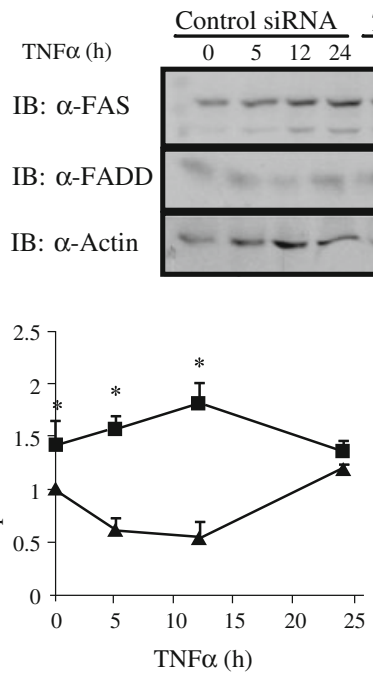

b

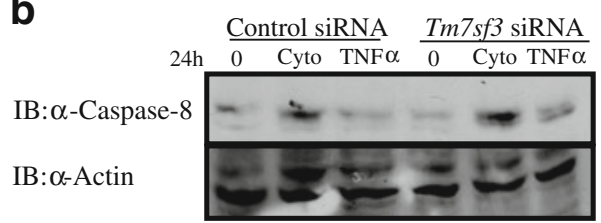

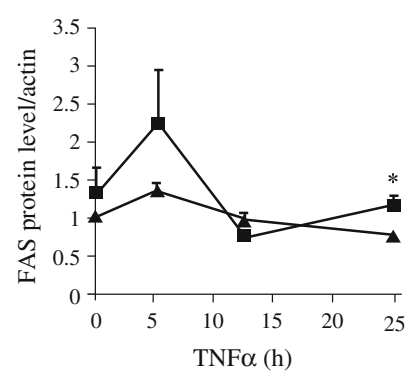

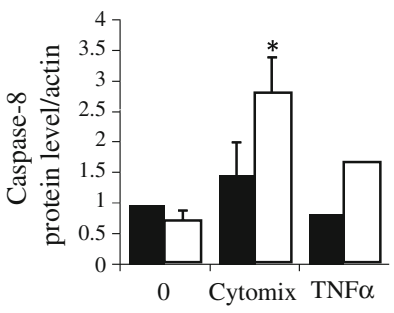

Fig. 5 Effects of TM7SF3 on the extrinsic death pathway. a MIN6 cells were transfected with non-targeting siRNAs or with $\operatorname{Tm} 7 s f 3$ siRNAs for $48 \mathrm{~h}$, before being treated with TNF $\alpha$ for the indicated times. Total cell extracts were resolved by SDS-PAGE and immunoblotted with FAS and FADD antibodies. Quantification of blots: triangles, non-targeting siRNAs; squares, $\operatorname{Tm} 7 s f 3$ siRNAs. b MIN6 cells were transfected with non-targeting siRNAs or with $T m 7 s f 3$

genes such as $\operatorname{Tm} 7 s f 3$ in regulating this process. The screens analysed caspase-3/7 activity and cellular reducing power, and revealed that cytokine-induced death of cultured MIN6 beta cells is a complex event involving apoptotic and necrotic elements, with a unique set of non-overlapping genes regulating each process. Our screens highlighted the relative importance of ubiquitination in cytokine-induced death, with ubiquitin ligases playing a major role in death induction and deubiquitinating enzymes promoting cell survival. The screens also underscored the involvement of protein kinases in the apoptotic process and of protein phosphatases in non-apoptotic (e.g. necrotic) cell death (Fig. 6a).

Several lines of evidence support the above conclusions. First, we showed that, in agreement with previous findings [3], the apoptotic pathway is fundamentally involved in beta cell death induced by cytokines [29-31]. A number of genes shown in our screens to be pro-apoptotic are established players in cytokine-induced death. These include IRF7 [32], CEBPB and $L M X 1 B$, the last two of which are encoding inducers of NF- $\mathrm{KB}$, a mediator of cytokine-triggered beta cell death [21,33].

Two molecular systems appear to play a key role in cytokine-induced beta cell apoptosis, one involving the ubiquitin system, and the other involving protein phosphorylation. We found that the family of ubiquitin ligases is enriched in pro-apoptotic genes, whereas the family of deubiquitinating enzymes [34] is enriched in anti-apoptotic genes (Fig. 6a). These observations are in
siRNAs for $48 \mathrm{~h}$, before being treated with TNF $\alpha$ or with Cytomix for $24 \mathrm{~h}$. Total cell extracts were resolved by SDS-PAGE and immunoblotted with the indicated antibodies. Quantification of blots: black bars, non-targeting siRNAs; white bars, $T m 7 s f 3$ siRNAs. Data represent mean \pm SEM of three (a) and two (b) independent experiments; ${ }^{*} p<0.05$ vs control (non-targeting) siRNA assayed under the same conditions

line with studies implicating ubiquitination as a regulator of central modules along the NF- $\mathrm{kB}$ signalling, a key pathway of cytokine-induced beta cell death [3]. These modules include degradation of I $k \mathrm{~B}$ (an NF- $\mathrm{kB}$ inhibitor) [35], processing of NF-KB precursors and activation of IKB kinase (IKK) [36]. The cellular inhibitor of apoptosis protein (cIAP) 1 and cIAP2 are also subjected to ubiquitin-induced degradation [37]. Conversely, the deubiquitinating enzymes, cylindromatosis (turban tumour syndrome) and A20, deubiquitinate and inactivate mediators of the NF- $\mathrm{KB}$ pathway, including receptor-interacting protein 1, TNF receptor-associated factor 6 and IKK $\gamma$ [36]. Hence, the main contribution of the deubiquitinating enzymes lies in their inactivation of the NF- $\mathrm{kB}$ pathway, thus inhibiting beta cell apoptosis. The second system that affects beta cell apoptosis is the kinases/phosphatases network. Indeed, many elements along the cytokineinduced apoptotic pathways are protein kinases such as IKK, JNK and p38.

Non-apoptotic pathways have also been implicated in the induction of beta cell death [16]. Cytokines trigger necrotic activity [38] by inhibiting aconitase (which reduces cellular ATP content) or by inducing nitric oxide production, which activates poly(ADP) ribose polymerase and $\mathrm{NAD}^{+}$depletion, thus leading to beta cell necrosis [7]. We found that protein phosphatases seem to be mainly involved in the induction of necrotic beta cell death, together with novel 'hits' such as general transcription factor IIH, polypeptide 1 , ST3 $\beta$-galactoside $\alpha$-2,3- 
Fig. 6 Cellular modalities involved in beta cell death. a Proposed model for the key cellular modalities involved in cytokine-induced beta cell death. b Model of genes inducing beta cell death according to the screens. ${ }^{a}$ Novel hits validated by deconvolution; ${ }^{b}$ novel hits validated by deconvolution and quantitative RT-PCR; upward arrows, pro-apoptotic proteins; downward arrows, antiapoptotic proteins a

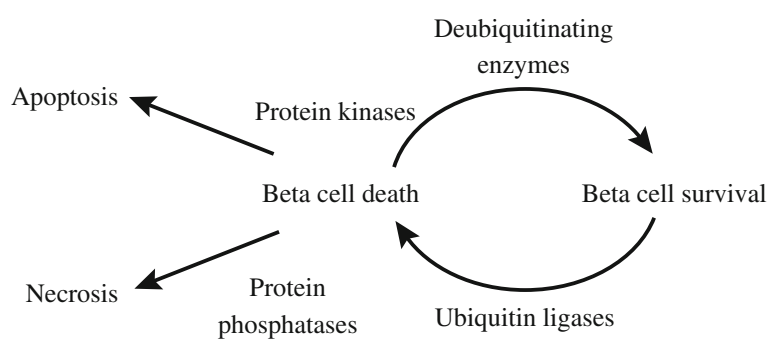

b

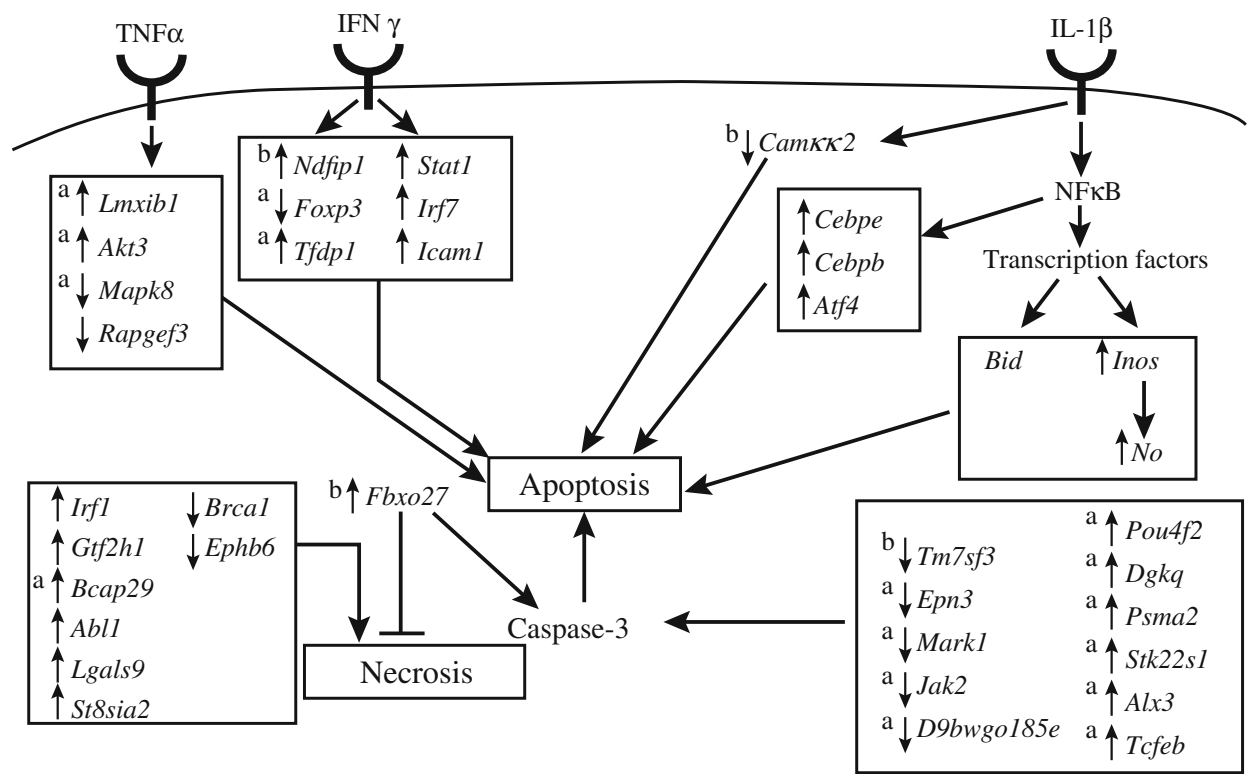

sialyltransferase 1 and anaplastic lymphoma kinase, which need to be further characterised.

An interesting outcome of the screens was the identification with high confidence of several novel pro-apoptotic 'hits', such as Gyk and Lmxlb (Table 1). Their role in mediating cytokine-induced death of beta cells remains to be determined. Others, like Foxp3 and Epn3, inhibit cytokine-induced beta cell death by as yet unknown mechanisms. Our high-confidence anti-apoptotic 'hits' also included Camkk2 and Jak2. Akt activation by calcium/ calmodulin-dependent protein kinase kinase 2 beta, inhibits IL-1 $\beta$-induced NF-kB activation [39]; Camkk2 can thus act as an anti-apoptotic gene in beta cells. Janus kinase 2, which phosphorylates signal transducer and activator of transcription 3, increases suppressor of cytokine signalling 3 production, thereby protecting pancreatic islets from cytokine-induced apoptosis [40].

One novel anti-apoptotic 'hit' revealed by the screen was $T m 7 s f 3$. It encodes a 7-transmembrane 'orphan' receptor [25] and is one of ten genes that were found in the $700 \mathrm{~kb}$ murine type 1 diabetes locus, Idd6.3 [41]. Our findings suggest that the main action of TM7SF3 is associated with maintaining cellular reducing power within physiological levels. It also acts to reduce the cellular content of proapoptotic proteins such as FAS, FADD and caspase-8; therefore its siRNAs increase the cellular content of these proteins. $T m 7 s f 3$ could therefore be considered a novel antiapoptotic gene. This is further illustrated by examining the alterations in cell morphology following treatment with $T m 7 s f 3$ siRNAs. Cells thus treated undergo apoptosis, characterised by cell swelling, rounding-up into 3D aggregates and perturbation of microtubules organisation, a characteristic feature of apoptotic cells. Because TM7SF3 acts to maintain cellular reducing power, it acts to increase GSIS, a process triggered by an increase in cellular ATP following glucose uptake. Hence, knockdown of $T m 7 s f 3$ is most likely to result in a reduction of cellular ATP and inhibition of GSIS, a process that is aggravated in the presence of cytokines. Consistent with this conclusion, inhibition of TM7SF3 expression by its siRNA significantly increased cell death of human islets induced by cytokines.

In summary, the above results enabled us to generate a signalling network of genes involved in cytokine-induced beta cell death (Fig. 6b). The network contains known genes, as well as novel, high-confidence candidates, whose contribution to beta cell death by apoptotic and non- 
apoptotic means is revealed in this study. These novel genes, including $\operatorname{Tm} 7 s f 3$, deserve further characterisation and may offer new targets for therapeutic intervention in type 1 diabetes and its adverse complications.

Acknowledgements We thank G. Yona for his helpful advice and technical assistance. We also thank J.I. Miazaki (Osaka University, Osaka, Japan) for providing MIN6 cells and S. Sampson (Bar Ilan University, Israel) for his critical review of the manuscript. This work was supported by research grants from The Juvenile Diabetes Research Foundation International, The Israel Science Foundation (grant number 759/09), D-CURE Israel, The Mitchel Kaplan Fund and the Minerva Foundation. Y. Zick is the incumbent of the Marte R. Gomez Professorial Chair and B. Geiger is the incumbent of the E. Neter Professorial chair of Cell and Tumor Biology. Human islets were provided through the Juvenile Diabetes Research Foundation award 31-2008-413 (ECIT Islet for Basic Research programme).

Contribution statement $\mathrm{AB}, \mathrm{RI}, \mathrm{IL}, \mathrm{YH}, \mathrm{TV}$ and HS-A contributed to conception and design of the experiments, data collection, data analysis interpretation and drafting of the article. BG and $\mathrm{YZ}$ contributed to conception and design of the experiments, data analysis and interpretation, as well as drafting of the article and revising it critically for important intellectual content. All authors gave final approval of the version to be published.

Duality of interest The authors declare that there is no duality of interest associated with this manuscript.

\section{References}

1. Hogan A, Pileggi A, Ricordi C (2008) Transplantation: current developments and future directions; the future of clinical islet transplantation as a cure for diabetes. Front Biosci 13:11921205

2. Mathis D, Vence L, Benoist C (2001) Beta-cell death during progression to diabetes. Nature 414:792-798

3. Cnop M, Welsh N, Jonas JC, Jorns A, Lenzen S, Eizirik DL (2005) Mechanisms of pancreatic $\beta$-cell death in type 1 and type 2 diabetes: many differences, few similarities. Diabetes 54(Suppl 2): S97-S107

4. Cadone MH, Salvesen GS, Widmann C, Johnson G, Frisch SM (1997) The regulation of anoikis: MEKK-1 activation requires cleavage by Caspases. Cell 90:315-323

5. Sarkar SA, Kutlu B, Velmurugan K et al (2009) Cytokinemediated induction of anti-apoptotic genes that are linked to nuclear factor kappa-B (NF-kappa B) signalling in human islets and in a mouse beta cell line. Diabetologia 52:1092-1101

6. Ortis F, Naamane N, Flamez D et al (2010) Cytokines interleukin1 beta and tumor necrosis factor-alpha regulate different transcriptional and alternative splicing networks in primary beta-cells. Diabetes 59:358-374

7. Eizirik DL, Mandrup-Poulsen T (2001) A choice of death-the signal-transduction of immune-mediated beta-cell apoptosis. Diabetologia 44:2115-2133

8. Riedl SJ, Shi Y (2004) Molecular mechanisms of caspase regulation during apoptosis. Nat Rev Mol Cell Biol 5:897-907

9. Ortis F, Pirot P, Naamane N et al (2008) Induction of nuclear factor-kappa B and its downstream genes by TNF-alpha and IL-1 beta has a pro-apoptotic role in pancreatic beta cells. Diabetologia 51:1213-1225
10. Degterev A, Hitomi J, Germscheid M et al (2008) Identification of RIP1 kinase as a specific cellular target of necrostatins. Nat Chem Biol 4:313-321

11. Kawasaki E, Abiru N, Eguchi K (2004) Prevention of type 1 diabetes: from the view point of beta cell damage. Diabetes Res Clin Pract 66(Suppl 1):S27-S32

12. Bernstein E, Caudy AA, Hammond SM, Hannon GJ (2001) Role for a bidentate ribonuclease in the initiation step of RNA interference. Nature 409:363-366

13. Ramadan N, Flockhart I, Booker M, Perrimon N, Mathey-Prevot B (2007) Design and implementation of high-throughput RNAi screens in cultured Drosophila cells. Nat Protoc 2:2245-2264

14. Berns K, Hijmans EM, Mullenders J et al (2004) A large-scale RNAi screen in human cells identifies new components of the p53 pathway. Nature 428:431-437

15. Miyazaki J, Araki K, Yamato E et al (1990) Establishment of a pancreatic beta cell line that retains glucose-inducible insulin secretion: special reference to expression of glucose transporter isoforms. Endocrinology 127:126-132

16. Collier JJ, Fueger PT, Hohmeier HE, Newgard CB (2006) Proand antiapoptotic proteins regulate apoptosis but do not protect against cytokine-mediated cytotoxicity in rat islets and beta-cell lines. Diabetes 55:1398-1406

17. Harvey AJ, Kind KL, Thompson JG (2002) REDOX regulation of early embryo development. Reproduction 123:479-486

18. Chen M, Yang Z, Wu R, Nadler JL (2002) Lisofylline, a novel antiinflammatory agent, protects pancreatic beta-cells from proinflammatory cytokine damage by promoting mitochondrial metabolism. Endocrinology 143:2341-2348

19. Eisinger-Mathason TS, Andrade J, Groehler AL et al (2008) Codependent functions of RSK2 and the apoptosis-promoting factor TIA-1 in stress granule assembly and cell survival. Mol Cell 31:722-736

20. Budhram-Mahadeo VS, Bowen S, Lee S et al (2006) Brn-3b enhances the pro-apoptotic effects of p53 but not its induction of cell cycle arrest by cooperating in trans-activation of bax expression. Nucleic Acids Res 34:6640-6652

21. Rascle A, Neumann T, Raschta AS et al (2009) The LIMhomeodomain transcription factor LMX1B regulates expression of NF-kappa B target genes. Exp Cell Res 315:76-96

22. Kim KA, Lee MS (2009) Recent progress in research on beta-cell apoptosis by cytokines. Front Biosci 14:657-664

23. Satoo K, Noda NN, Kumeta H et al (2009) The structure of Atg4B-LC3 complex reveals the mechanism of LC3 processing and delipidation during autophagy. EMBO J 28:1341-1350

24. Kroemer G, Galluzzi L, Vandenabeele P et al (2009) Classification of cell death: recommendations of the Nomenclature Committee on Cell Death 2009. Cell Death Differ 16:3-11

25. Akashi H, Han HJ, Iizaka M, Nakamura Y (2000) Growthsuppressive effect of non-steroidal anti-inflammatory drugs on 11 colon-cancer cell lines and fluorescence differential display of genes whose expression is influenced by sulindac. Int $\mathrm{J}$ Cancer $88: 873-880$

26. Bershadsky A, Chausovsky A, Becker E, Lyubimova A, Geiger B (1996) Involvement of microtubules in the control of adhesiondependent signal transduction. Curr Biol 6:1279-1289

27. Howell SL (1984) The mechanism of insulin secretion. Diabetologia 26:319-327

28. Wang L, Du F, Wang X (2008) TNF-alpha induces two distinct caspase-8 activation pathways. Cell 133:693-703

29. Giannoukakis N, Rudert WA, Trucco M, Robbins PD (2000) Protection of human islets from the effects of interleukin-1beta by adenoviral gene transfer of an Ikappa B repressor. J Biol Chem 275:36509-36513

30. Jambal P, Masterson S, Nesterova A et al (2003) Cytokinemediated down-regulation of the transcription factor cAMP- 
response element-binding protein in pancreatic beta-cells. J Biol Chem 278:23055-23065

31. Emamaullee JA, Shapiro AM (2006) Interventional strategies to prevent $\beta$-cell apoptosis in islet transplantation. Diabetes 55:1907-1914

32. Ning S, Campos AD, Darnay BG, Bentz GL, Pagano JS (2008) TRAF6 and the three C-terminal lysine sites on IRF7 are required for its ubiquitination-mediated activation by the tumor necrosis factor receptor family member latent membrane protein 1 . Mol Cell Biol 28:6536-6546

33. Chen J, Zhao M, Rao R, Inoue H, Hao CM (2005) C/EBP $\beta$ and its binding element are required for NFKB-induced COX2 expression following hypertonic stress. J Biol Chem 280:1635416359

34. Shin JM, Yoo KJ, Kim MS, Kim D, Baek KH (2006) Hyaluronan- and RNA-binding deubiquitinating enzymes of USP17 family members associated with cell viability. BMC Genomics 7:292

35. Karin M (1999) The beginning of the end: IkappaB kinase (IKK) and NF-kappaB activation. J Biol Chem 274:27339-27342
36. Chen ZJ (2005) Ubiquitin signalling in the NF-kappaB pathway. Nat Cell Biol 7:758-765

37. Varfolomeev E, Blankenship JW, Wayson SM et al (2007) IAP antagonists induce autoubiquitination of c-IAPs, NF-kappaB activation, and TNFalpha-dependent apoptosis. Cell 131:669-681

38. Kroemer G, Martin SJ (2005) Caspase-independent cell death. Nat Med 11:725-730

39. Chen BC, Wu WT, Ho FM, Lin WW (2002) Inhibition of interleukin-1beta-induced NF-kappa B activation by calcium/ calmodulin-dependent protein kinase kinase occurs through Akt activation associated with interleukin-1 receptor-associated kinase phosphorylation and uncoupling of MyD88. J Biol Chem 277:24169-24179

40. Rezende LF, Vieira AS, Negro A, Langone F, Boschero AC (2009) Ciliary neurotrophic factor (CNTF) signals through STAT3SOCS3 pathway and protects rat pancreatic islets from cytokineinduced apoptosis. Cytokine 46:65-71

41. Hung MS, Avner P, Rogner UC (2006) Identification of the transcription factor ARNTL2 as a candidate gene for the type 1 diabetes locus Idd6. Hum Mol Genet 15:2732-2742 